\title{
Biomechanical evaluation of an arthroscopic transosseous repair as a revision option for failed rotator cuff surgery
}

Felix Dyrna ${ }^{1} \mathbb{D}$, Andreas Voss', Leo Pauzenberger², Elifho Obopilwe², Augustus D. Mazzocca², Alessandro Castagna ${ }^{3}$ and Cory Edgar ${ }^{2^{*}}$

\begin{abstract}
Background: The number of revision rotator cuff cases is increasing. The literature is lacking guidance or biomechanical evaluation for fixation strength in a revision case scenario. Therefore, the aim of the study was to provide biomechanical data investigating primary fixation strength of a transosseous technique after anchor pullout failure of a single row reconstruction. It was hypothesized that an arthroscopic transosseous repair system as a procedure for rotator cuff revisions is providing equivalent stability compared to a primary single row suture anchor fixation due to change of fixation site.

Methods: Eight matched pairs $(n=16)$ of fresh frozen human shoulders were tested. The paired specimen shoulders were randomly divided into two repair groups (A single row and B primary transosseous repair). The potted specimens were mounted onto the Servohydraulic test system. Both groups were tested under cyclic loading followed by load to failure testing. Suture anchor repair shoulders (group A) that were tested to failure underwent a revision transosseous repair and were subsequently tested again using the same setup and protocol (group C).
\end{abstract}

Results: The mean native footprint areas did not show a significant difference between groups. The reconstructed footprint area showed a significantly greater coverage in favor of the transosseous repair. Ultimate load to failure of reconstructions with the primary anchor fixation $(344.73 \mathrm{~N} \pm 63.19)$ and the primary transosseous device $(375.36 \mathrm{~N}$ \pm 70.27 ) was not significantly higher compared to the revision repair $(332.19 \mathrm{~N} \pm 119.01 p=0.45, p=0.53$ ).

Conclusion: The tested transosseous anchor device is a suitable option to widely used suture anchors, providing equivalent fixation properties even in a revision case scenario.

Level of evidence: Basic Science Study, Biomechanics.

Keywords: Rotator cuff, Cuff repair, Revision, Transosseous repair, Arthroscopic repair

\footnotetext{
*Correspondence: coedgar@uchc.edu

${ }^{2}$ Department of Orthopaedic Surgery, University of Connecticut, Farmington,

CT, USA

Full list of author information is available at the end of the article
}

(c) The Author(s). 2018 Open Access This article is distributed under the terms of the Creative Commons Attribution 4.0 International License (http://creativecommons.org/licenses/by/4.0/), which permits unrestricted use, distribution, and reproduction in any medium, provided you give appropriate credit to the original author(s) and the source, provide a link to the Creative Commons license, and indicate if changes were made. The Creative Commons Public Domain Dedication waiver (http://creativecommons.org/publicdomain/zero/1.0/) applies to the data made available in this article, unless otherwise stated. 


\section{Background}

Despite promising results in biomechanical studies, the clinical re-tear rate of rotator cuff repairs is still high. In the literature the incidence of recurrences has been reported of over $30 \%$ for small injuries [1,2] and may extend to $90 \%$ in cases of massive tears [3-6]. Reasons for failure of reconstruction are variegated [7]. Rotator cuff revision surgery is always challenging and results are often inferior to primary repairs $[8,9]$. Arthroscopic repair may present advantages compared to historical open transosseous repairs, such as minimal infraction of the deltoid muscle, the ability to dynamically inspect the entire glenohumeral joint for associated pathologies, characterize tear patterns, and decrease postoperative pain and stiffness $[10,11]$.

The quest for improved methods of rotator cuff repair led to the development of all-arthroscopic transosseous rotator cuff repair techniques [12], which could potentially combine the biomechanical strengths of open transosseous repairs with all of the advantages of arthroscopic techniques. Recent studies, that have compared traditional transosseous equivalent techniques with modern single- and double-row anchor-based configurations, showed superior load to failure strength in transosseous repairs $[13,14]$.

The purpose of the present study was to compare displacement under cyclic loading, load-to-failure, and footprint coverage of rotator cuff reconstructions performed with a new, all-arthroscopic transosseous repair (TOR) system and an established single row suture anchor repair configuration, as (a) a primary repair technique and (b) in case of failed anchor based initial repair. Our primary hypothesis was that an arthroscopic transosseous repair system as a procedure for rotator cuff revisions is providing equivalent stability compared to a primary single row suture anchor fixation due to change of fixation site.

\section{Methods}

Preparation

The test involved the use of eight matched pairs $(n=16)$ fresh frozen human shoulders without macroscopic evidence of rotator cuff pathology. The mean age of specimens was 69,9 years with a range from 74 to 66 years including 8 female and 8 male specimens. All specimens were obtained from Medcure Inc. (Portland, OR). The study was reported via Human Research Determination Form to the institutional review board (IRB) of the University of Connecticut and it was documented, that no IRB approval was required (de-identified specimen do not constitute human subjects research). Specimens were thawed for $24 \mathrm{~h}$ at room temperature prior to dissection. Bone Density via micro-CT (Lunar DXE, Madison, Wis) was performed to ensure consistent bone quality among tested specimens at the greater tuberosity in a consistent manner at a $1 \times 1-\mathrm{cm}$ area.

Selected specimens were prepared by removing all soft tissues, but leaving the supraspinatus, infraspinatus, and teres minor tendons intact. The native supraspinatus footprint was measured with digital calipers for linear measurements (Absolute Digimatic; Mitutoyo, Kawasaki, Japan). While the supraspinatus muscle was held in a superior direction, the width of the footprint at its greatest dimension was measured by placing 1 limb of the digital caliper precisely on the articular edge of the intact supraspinatus tendon and the other arm of the caliper on the lateral bursal-side edge of the insertion. The anterior and posterior insertions of the supraspinatus were measured likewise. This procedure allowed an estimated contact area to be calculated. Although the supraspinatus footprint does not represent a true rectangle, these measurements provided a consistent means to compare the contact areas of the intact and repaired supraspinatus tendons. To ensure correct measurements, results were compared with a measured area using a MicroScribe digitizer (Immersion, San Jose, CA) by lifting the supraspinatus muscle and tracing the outline of the attachment [15], before creating the tear. The same investigator performed all footprint measurements. The next step involved the sharp dissection of the supraspinatus tendon from its footprint to create a uniform $20 \times 10 \mathrm{~mm}$ full-thickness tendon tissue defect approximately $5 \mathrm{~mm}$ proximally to the humeral insertion of the tendon, as previously described [16].

Prior to the repair the footprint area was macroscopically completely decorticated to mimic a revision case scenario and weaken the bone quality. The paired specimen shoulders were randomly divided into two groups. A total of 8 specimens per group were tested.

\section{Repair configurations \\ Group A Single Row}

The single-row repair was performed using two double loaded titanium corkscrew anchors (Arthrex, Naples, FL) placed lateral to the cartilage margin in the greater tuberosity $12 \mathrm{~mm}$ apart. The anchors were placed at the "dead man's angle" to maximize pullout strength [17]. Both strands of No. 2 FiberWire (Arthrex, Naples, FL) in each anchor were then shuttled in a typical fashion using a Suture Lasso (Fig. 1a). Knot tying was performed in arthroscopic fashion using a standard knot pusher with overhand throws, alternating half hitches and posts to maximize loop and knot security [18].

\section{Group B primary TOR}

The transosseous repair was performed with the Sharc-FT ${ }^{\bullet}$ system (NCS Lab, Medical Devices Factory), 


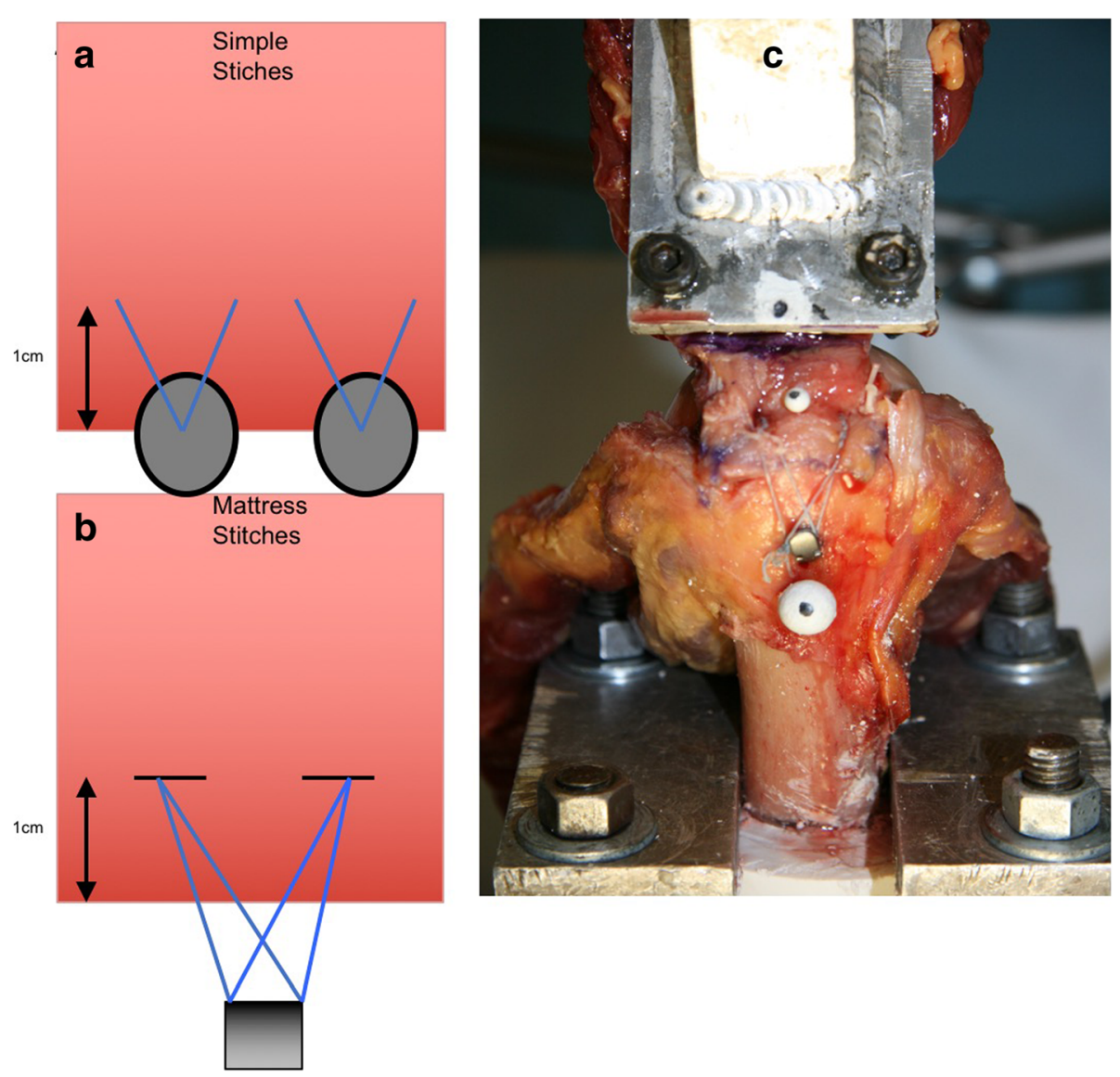

Fig. 1 shows (a) single row repair, (b) TOR with Sharc-FT, (c) TOR repair final set-up

which is based on diverging canals created by a drill guide the Taylor stitcher ${ }^{\bullet}$ (NCS Lab, Medical Devices Factory) through the great tuberosity of the humerus consisting of one lateral entering and - depending on tear size - multiple medial exit tunnels. The transosseous configuration involved the creation of a lateral entry hole centered laterally from the lesion and two medial exit holes, one anterior and one posterior with a distance of $10-12 \mathrm{~mm}$ in between. Therefore, the first medial exit tunnel, a $2 \mathrm{~mm}$ hole in the ventral third of the footprint was punched with the guidance of the Taylor stitcher ${ }^{\circ}$ device (NCS Lab, Medical Devices Factory). Furthermore, this guide was used to place the lateral $2 \mathrm{~mm}$ hole $3 \mathrm{~cm}$ from the greater tuberosity edge. The posterior medial exit tunnel was created in the same manner while keeping the guide in the lateral exit hole. Once the canals were placed, the Sharc-FT (NCS Lab, Medical Devices Factory) device preloaded with two internal sutures was used to shuttle the definite sutures, No. 2 FiberWire (Arthrex, Naples, FL). These sutures were arranged to obtain two mattress stitches over the tendon (Fig. 1b), then the four suture limbs were used to close the suture loop passing them through the eyelet on the device to function as a second row increasing the footprint coverage and area of compression [19].

\section{Group C revision Transosseous repair with Sharc-FT}

After load-to-failure testing of the single-row group, shoulders, which showed a sufficiently intact footprint and tendon after initial testing, underwent a revision procedure with the Sharc-FT(R) (NCS Lab, Medical Devices Factory) system following the before mentioned technique. Hence, comparing transosseous refixation with this device as a suitable revision technique. Again the aiming device (Taylor stitcher) was used to place as previously descripted the two converging tunnels at the spot of the removed anchors and the revision repair was completed.

\section{Biomechanical testing}

All samples were tested in air at room temperature. The humerus was cut $20 \mathrm{~cm}$ from the joint, centered in a polyvinylchloride pipe, and potted with plaster of Paris. The potted cadaveric specimen was then mounted on the testing apparatus with the tendons oriented in line with the anatomic pull of the myo-tendinous unit The medial portion of the supraspinatus tendon was 
freeze-clamped and attached to a MTS, Eden Prairie, $\mathrm{MN})$ as described in previous studies $[18,20]$. In this setup, the specimens were tested under cyclic load, while measuring gap formation. To quantify gap formation one marker was placed laterally below the greater tuberosity and one in line centric on the musculotendinous junction of the supraspinatus.

A video digitizing system that involved video recording and computer digitization of the markers, creation of centroids representing the center of the markers, and the calculation of distances between them with MaxTRAQ 2D (Innovision Systems, Inc., Columbiaville, Michigan) software was used (Fig. 1c).

After a preload phase with $10 \mathrm{~N}$ for $1 \mathrm{~min}$ and ten initial cycles, the load was set to change from a minimum value of $10 \mathrm{~N}$ to a maximum of $180 \mathrm{~N}$ at a velocity of $33 \mathrm{~mm} / \mathrm{s}$ [21-24] for a total of 200 cycles [21, 25-27]. In the event of gap formation greater than $5 \mathrm{~mm}$, the test was stopped and the number of completed cycles was noted. After a preload phase samples that reached 200 cycles with a gap variation less than $5 \mathrm{~mm}$ were then tested to load-of-failure at a rate of $33 \mathrm{~mm} / \mathrm{s}$.

Shoulders from the anchor repair group A that were tested to failure underwent a revision transosseous repair and were subsequently tested regarding gap formation under cyclic loading and load-to-failure using the same setup and protocol group $C$.

\section{Statistical analyses}

Given the exploratory nature of this study, an a priori power analysis was difficult. We anticipated a medium effect size (30-45\% difference in load to failure or about $100 \mathrm{~N}$ ) between the primary suture anchor and revision transosseous repair with an estimated variability of $\pm 50 \mathrm{~N}$. A sample size of 6 per group will provide $80 \%$ power to detect a difference of $100 \mathrm{~N}$ an alpha value of 0.02 between the repair groups. Descriptive statistics were calculated using mean and standard deviation for the repair groups. Both independent and pair t test were used to compare differences in load to failure, slope, displacement, and footprint reconstruction. The revision trans-osseous repair group included the same shoulders tested in primary suture group creating dependency within the data which necessitated the use of separate $t$ tests as opposes to a global ANOVA. To avoid inflating our Type I error rate, a Bonferroni adjusted $p$ value of 0.02 was used as the threshold for statistical significance. All statistical analysis was performed using Stata 12 (StataCorp. 2011. Stata Statistical Software: Release 12. College Station, TX: StataCorp LP.).

\section{Results}

The results for footprint reconstruction, displacement during cyclic loading, load to failure and stiffness were summarized in Fig. 2 a-d. The bone quality (BMD) was measured for each specimen and did not differ between groups (group A mean $0.361 \mathrm{~g} / \mathrm{cm}^{2} \pm 0.05$ and group B $\left.0.435 \mathrm{~g} / \mathrm{cm}^{2} \pm 0.09 p=0.094\right)$.

\section{Footprint coverage}

The mean native footprint areas in group $\mathbf{A}$ $264.91 \mathrm{~mm}^{2} \pm 103.12$, and group B $307.68 \mathrm{~mm}^{2} \pm 116.13$ did not show a significant difference $p=0.48$. Since group $\mathbf{C}$ includes the same specimens as group $\mathbf{A}$ the native footprints are equal. The covered footprint areas after the repair in group A $192.48 \mathrm{~mm}^{2} \pm 59.55$, group B $246.25 \mathrm{~mm}^{2} \pm 61.16$, and group C $259.93 \mathrm{~mm}^{2} \pm 43.28$ were compared A vs. B $p=0.01$ and $\mathrm{A}$ vs. $\mathrm{C} p=0.12$ in favor of the transosseous repair.

\section{Displacement}

200 cycles of repetitive motion at time zero resulted in no significant differences between the three groups (A vs. $\mathrm{B} p=0.57$, A vs. $\mathrm{C} p=0.98$ and $\mathrm{B}$ vs. $\mathrm{C} p=0.47$ ). Mean displacement between the groups was not significant and represented in Fig. 2b An overall displacement over $5 \mathrm{~mm}$ was considered as a failure. This occurred once in group $\mathbf{A}$ and group $\mathbf{B}$ as the anchor already pulled out but not in group C. (Fig. 2b).

\section{Ultimate Load to Failure}

Ultimate load to failure of reconstructions with the primary anchor fixation (344.73 $\mathrm{N} \pm 63.19$ group A) and the primary Sharc-FT device (375.36 $\mathrm{N} \pm 70.27$ group B) was not significantly higher compared to the revision repair (332.19 $\mathrm{N} \pm 119.01$ group C, A vs. B $p=0.45$, A vs. C $p=0.53$ ). There were also no significant differences comparing the slope of the constructs, and yield loads. (Fig. 2c, d). The failure modes were 5/8 (63\%) anchor pullouts and $3 / 8(37 \%)$ suture pull through in group $\mathrm{A}$. For group $B$ the main failure was suture cutting through tendon 5/8 (63\%) and 3/8 (37\%) bony suture pullouts. Group C showed 6/8 (75\%) anchor pullouts and 2/8 (25\%) suture tendon interface failures.

\section{Discussion}

The purpose of this study was to evaluate the load to failure, stiffness, displacement under cyclic loading, and footprint reconstruction of two different fixations at time zero. The number of suture passages through the tendon was equal $(n=4)$ between the groups to focus on bony fixation strength. Generated results did confirm the original hypothesis that the transosseous fixation technique restores similar fixation strength in a revision case scenario compared to primary single row anchor fixation. We found no significant difference in the biomechanical properties of the compared techniques. The rotator cuff repair represents one of the most frequently 

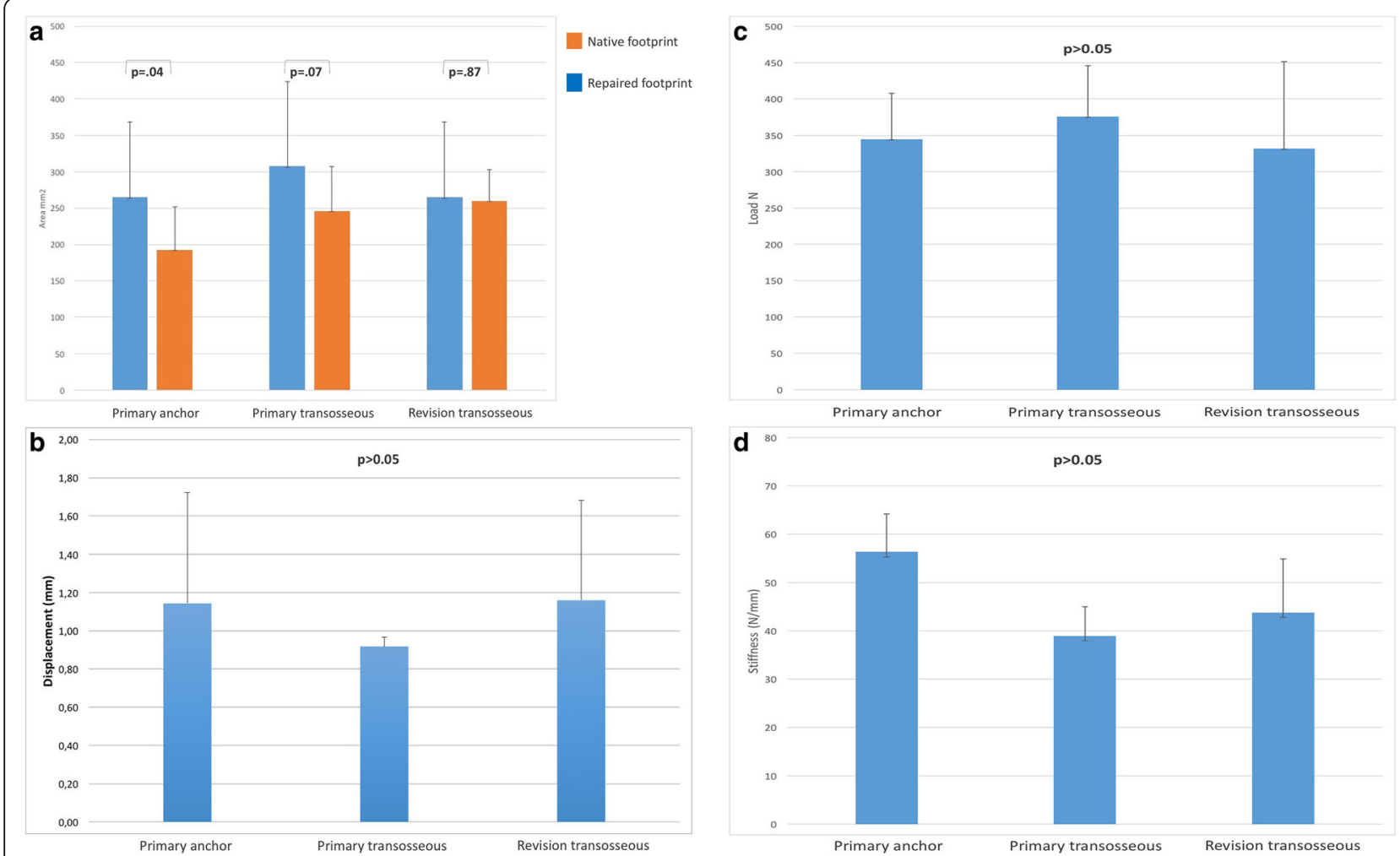

Fig. 2 (a) displaces the native and repaired footprint data for each repair technique, (b) represents displacement data after cyclic loading, (c, d) shows ultimate load to failure results and stiffness of the repair constructs

performed orthopedic surgical intervention [28]. Hence of numerous technological innovations, the re-tear rate is still unsatisfactory high $[3,4,7,10]$.With a raising number of surgeons performing their arthroscopic rotator cuff repairs with suture anchors our attention has been placed on a possible revision fixation technique. To our mind the TOR serves as a reasonable option to fulfill this task preserving similar biomechanical parameters by changing the fixation site. McLaughlin was the first one describing a transosseous rotator cuff repair back in 1944 [29]. Since then a progress of technological improvements established a save and reproducible technique that can even be performed arthroscopically [19]. According to Gerber et al. [30], in theory a perfect repair should combined high fixation strength, minimal gap formation, and sufficient mechanical stability for tendon-bone healing. The existing literature consist of studies demonstrating that transosseous techniques have equivalent biomechanical properties relative to suture anchor fixation [21, 31-33]. Lately, Behrens et al. [34] showed that traditional transosseous suture construct without anchors can even provide an initial fixation strength equivalent to suture bridge repairs. The stress load is distributed wider in the bone tunnel of the transosseous repair, whereas in suture anchor repair, resulting in less local stress loads [35]. In addition, the pressure is more homogenously assigned in transosseous sutures in opposite to the anchor repair, where high values may be a risk factor for ischemic tissue damage [36]. To evaluate the Gap-formation and displacement two optical markers were positioned centric on either side of the repair as shown in Fig. 1c. This position was chosen to analyze the average displacement since Tashjian et al. [32] found that gapping significantly increased from the anterior to posterior region. The gap formation observed in our three groups did not differ significantly and approached similar values as previously reported in literature [34]. Hence, we had two failures in groups A and $\mathrm{B}$ and no failure in group $\mathrm{C}$ during cyclic loading due to anchor pull outs caused by weak bone quality. The bone quality (BMD) was measured for each specimen and did not differ between groups (group A mean $0.361 \mathrm{~g} / \mathrm{cm}^{2} \pm$ 0.05 and group B $\left.0.435 \mathrm{~g} / \mathrm{cm}^{2} \pm 0.09 p=0.094\right)$. The macroscopically decortication was performed after the BMD measuring and prior to the repairs to mimic poor bone quality. In regarding to Tingart et al. [37] who published that a successful rotator cuff repair highly depends the bone density at the greater tuberosity we expected to weaken our primary suture anchor repair by this decortication process but we still reached comparable anchor pull out values compared to the literature [31]. Pointing out that we may did not weaken the bone 
significantly even if macroscopically a full removal of cortical bone was performed. Pointing out that the suture anchors mainly rely on the trabecular, not the cortical bone [38]. During our ultimate load to failure testing, anchor pullout was the main failure mode in all groups A-C. The most interesting factor is that we were able to show that even after pulling out a suture anchor repair the subsequent implanted TOR was not significantly weaker than the initial anchor nor the initial TOR. Leading to the assumption that after a failed single row anchor repair the TOR is a possible revision option.

Knowing the initial strength characteristics of different rotator cuff repair techniques may influence surgical decision-making and may ultimately improve patient outcomes. Traditionally, the TOR technique was performed open and later modified to mini-open procedures. Apreleva et al. [39] demonstrated that TOR provides superior supraspinatus footprint coverage compared to suture anchor systems. Similar results were found in our study comparing a transosseous repair with a single row suture anchor.. The primary limitation of this study is that it is an in-vitro study only incorporating fixation strength at time point zero and a small sample size. The results cannot be extrapolated to the potential impact of tendon healing. Further the study specimens had intact rotator cuff tendons that do not replicate normal tendon and joint degeneration. We also created an isolated supraspinatus defect, which does not necessarily replicate the clinical situation. We try to include specimens with comparable age and bone quality. Lastly, the repairs in our study were performed under the ideal condition of being open procedures. Finally, the results of our study suggest that the initial fixation strength of single row suture anchor and TOR for rotator cuff repair at time point zero with comparable tendon and bone is equivalent. Both reconstructions prevented Gap-formation and early failure but preserved good footprint coverage. The revision TOR proved its biomechanical properties to be fully capable as an option for primary and secondary repairs. The clinical relevance of this study showed that there is no difference when comparing a current used arthroscopic single row suture anchor repair with a novel arthroscopic transosseous technique, supporting its use as a revision option.

\section{Conclusion}

The tested transosseous anchor device is a suitable option to widely used suture anchors, providing equivalent fixation properties even in a revision case scenario for single row reconstructions.

\section{Abbreviations}

ANOVA: Analysis of variance; BMD: Bone mineral density; TOR: Transosseous repair

\section{Funding}

The University of Connecticut Health Center / UConn Musculoskeletal Institute has received direct funding and material support for this study from NCS Lab s.r.l. (Carpi, Italia). The company had no influence on study design, data collection, interpretation of the results, or the final manuscript. Augustus D. Mazzocca has received research support from Arthrex, Inc., Naples, Florida.

This work was supported by the German Research Foundation (DFG) and the Technische Universität München within the funding programme Open Access Publishing

\section{Availability of data and materials}

The datasets generated and analyzed during the current study are not publicly available, but are available as de-identified data sheet from the corresponding author on reasonable request.

\section{Consent to publish}

No consent for publication was needed, because of the de-identified data.

\section{Authors' contributions}

Felix Dyrna, M.D. Principle investigator, data analysis, biomechanical testing. Andreas Voss, M.D. Biomechanical testing and data analysis.

Leo Pauzenberger, M.D. Revising the work critically for important intellectual content.

Elifho Obopilwe, M.S. Biomechanical support for testing and setup.

Augustus D. Mazzocca Data analysis, native language corrections.

M.S., M.D.

Alessandro Castagna M.D. Major contribution to discussion.

Cory Edga M.D., Ph.D. Surgical procedure and final approval of manuscript to be published.

The authors attest that the manuscript has been read and approved by all authors, and each author believes that the manuscript represents honest work.

Ethics approval and consent to participate

The biomechanics portion of the above study was conducted using only deidentified cadaveric specimens and is therefore not considered human subjects research. The study was reported via Human Research Determination Form to the institutional review board (IRB) of the University of Connecticut and it was documented, that no IRB approval was required While this form does not require IRB review, we have confirmed with our IRB that projects conducted in our biomechanics laboratory that utilize de-identified specimens does not constitute human subjects research and therefore no review required. No consent was required for the biomechanical study.

\section{Competing Interests}

The following authors declare that they have no competing interests: AV, LP, $\mathrm{EO}, \mathrm{AC}$.

The following authors declare that they have competing interests:

- FD received finical support from the German Research Foundation (DFG) and the Technische Universität München for publication of this manuscript (processing fee).

- ADM received research funding from Arthrex Inc., (Naples, FI, USA).

- CE received direct funding and material support for this study from NCS Lab s.r.l. (Carpi, Italia).

None of the authors have non-financial competing interests.

\section{Publisher's Note}

Springer Nature remains neutral with regard to jurisdictional claims in published maps and institutional affiliations.

\section{Author details}

${ }^{1}$ Department of Orthopaedic Sports Medicine, Technical University, Munich Germany. ${ }^{2}$ Department of Orthopaedic Surgery, University of Connecticut, Farmington, CT, USA. ${ }^{3}$ Department of Shoulder and Elbow IRCCS Humanitas Institute, Milan, Italy. 
Received: 8 January 2018 Accepted: 15 May 2018

Published online: 19 July 2018

\section{References}

1. Gazielly DF, Gleyze P, Montagnon C. Functional and anatomical results after rotator cuff repair. Clin Orthop Relat Res 1994;\&NA:-43???53.

2. Boileau P, Brassart N, Watkinson DJ, Carles M, Hatzidakis AM, Krishnan SG. Arthroscopic repair of full-thickness tears of the supraspinatus: does the tendon really heal? J Bone Joint Surg Am. The American Orthopedic Association. 2005;87:1229-40

3. Galatz LM, Ball CM, Teefey SA, Middleton WD, Yamaguchi K. The outcome and repair integrity of completely arthroscopically repaired large and massive rotator cuff tears. J Bone Joint Surg. 2004;86-A:219-24.

4. Heuberer PR, Smolen D, Pauzenberger L, Plachel F, Salem S, Laky B, et al. Longitudinal long-term magnetic resonance imaging and clinical follow-up after single-row arthroscopic rotator cuff repair: clinical superiority of structural tendon integrity. Am J Sports Med. 2017;45:1283-8.

5. Charousset C, Grimberg J, Duranthon L-D, Bellaiche L, Petrover D. Can a double-row anchorage technique improve tendon healing in arthroscopic rotator cuff repair?: a prospective, nonrandomized, comparative study of double-row and single-row anchorage techniques with computed tomographic arthrography tendon healing assessment. Am J Sports Med. 2007;35:1247-53.

6. Park J-Y, Siti HT, Keum J-S, Moon S-G, Oh K-S. Does an arthroscopic suture bridge technique maintain repair integrity?: a serial evaluation by ultrasonography. Clin Orthop Relat Res. Springer-Verlag. 2010;468:1578-87.

7. Spennacchio P, Banfi G, Cucchi D, D'Ambrosi R, Cabitza P, Randelli P. Long term outcome after arthroscopic rotator cuff treatment. Knee Surg Sports Traumatol Arthrosc. Springer Berlin Heidelberg. 2015;23:523-9.

8. Kilinc AS, Giarreti R, Diaz L, Sauzières P, Valenti P. Recurrent rotator cuff tears: an evaluation of open re-surgery results. Eur J Orthop Surg Traumatol. 2010;20:373-80.

9. Parnes N, DeFranco M, Wells JH, Higgins LD, Warner JJP. Complications after arthroscopic revision rotator cuff repair. Arthroscopy. Elsevier. 2013; 29:1479-86.

10. Piasecki DP, Verma NN, Nho SJ, Bhatia S, Boniquit N, Cole BJ, et al. Outcomes after arthroscopic revision rotator cuff repair. Am Orthop Soc Sports Med. 2010;38:40-6.

11. Wilson F, Hinov V, Adams $G$. Arthroscopic repair of full-thickness tears of the rotator cuff: 2- to 14-year follow-up. Arthroscopy. 2002;18:136-44.

12. Garofalo R, Castagna A, Borroni M, Krishnan SG. Arthroscopic transosseous (anchorless) rotator cuff repair. Knee Surg Sports Traumatol Arthrosc. 2012; 20:1031-5.

13. Lavery KP, Rasmussen JF, Dhawan A. Arthroscopic transosseous-equivalent rotator cuff repair. Arthrosc Tech. 2013;2:e183-5.

14. Lee TQ. Current biomechanical concepts for rotator cuff repair. Clin Orthop Surg. 2013;5:89-97.

15. Curtis AS, Burbank KM, Tierney JJ, Scheller AD, Curran AR. The insertional footprint of the rotator cuff: an anatomic study. Arthroscopy. 2006;22:603609.e1.

16. Lee S, Mahar A, Bynum K, Pedowitz R. Biomechanical comparison of bioabsorbable sutureless screw anchor versus suture anchor fixation for rotator cuff repair. Arthroscopy. 2005;21:43-7.

17. Burkhart SS. Reprint of: the deadman theory of suture anchors: observations along a South Texas fence line. Arthroscopy. 2014;30:895-9.

18. Mazzocca AD, Millett PJ, Guanche CA, Santangelo SA, Arciero RA. Arthroscopic single-row versus double-row suture anchor rotator cuff repair. Am J Sports Med. 2005;33:1861-8.

19. Baudi P, Rasia Dani E, Campochiaro G, Rebuzzi M, Serafini F, Catani F. The rotator cuff tear repair with a new arthroscopic transosseous system: the Sharc-FT( $\left.{ }^{\oplus}\right)$. Musculoskelet Surg Springer Milan. 2013;97(Suppl 1):57-61.

20. Beitzel K, Chowaniec DM, McCarthy MB, Cote MP, Russell RP, Obopilwe E, et al. Stability of double-row rotator cuff repair is not adversely affected by scaffold interposition between tendon and bone. Am J Sports Med. 2012;40: 1148-54.

21. Bisson L, Manohar LM. A biomechanical comparison of transosseous-suture anchor and suture bridge rotator cuff repairs in cadavers. Am J Sports Med. 2009;37:1991-5.

22. Burkhart SS, Johnson TC, Wirth MA, Athanasiou KA. Cyclic loading of transosseous rotator cuff repairs: tension overload as a possible cause of failure. Arthroscopy. 1997;13:172-6.
23. Meier SW, Meier JD. The effect of double-row fixation on initial repair strength in rotator cuff repair: a biomechanical study. Arthroscopy. 2006;22: 1168-73.

24. Zheng N, Harris HW, Andrews JR. Failure analysis of rotator cuff repair: a comparison of three double-row techniques. J Bone Joint Surg Am. 2008;90: 1034-42.

25. Kim DH, ElAttrache NS, Tibone JE, Jun B-J, DeLaMora SN, Kvitne RS, et al. Biomechanical comparison of a single-row versus double-row suture anchor technique for rotator cuff repair. Am J Sports Med. 2006:34:407-14.

26. Barber FA, Drew OR. A biomechanical comparison of tendon-bone interface motion and cyclic loading between single-row, triple-loaded cuff repairs and double-row, suture-tape cuff repairs using biocomposite anchors. Arthroscopy. 2012;28:1197-205.

27. Anderl W, Heuberer PR, Laky B, Kriegleder B, Reihsner R, Eberhardsteiner J. Superiority of bridging techniques with medial fixation on initial strength. Knee Surg Sports Traumatol Arthrosc. 2012;20:2559-66.

28. Iyengar JJ, Samagh SP, Schairer W, Singh G, Valone FH III, Feeley BT. Current trends in rotator cuff repair: surgical technique, setting, and cost. Arthroscopy. 2014;30:284-8.

29. McLaughlin HL. Lesions of the musculotendinous cuff of the shoulder. The exposure and treatment of tears with retraction. 1944. Clin Orthop Relat Res. 1994:304:3-9.

30. Gerber C, Schneeberger AG, Beck M, Schlegel U. Mechanical strength of repairs of the rotator cuff. J Bone Joint Surg Br. 1994;76:371-80.

31. Pietschmann MF, Fröhlich V, Ficklscherer A, Hausdorf J, Utzschneider S, Jansson $V$, et al. Pullout strength of suture anchors in comparison with transosseous sutures for rotator cuff repair. Knee Surg Sports Traumatol Arthrosc. Springer-Verlag. 2008;16:504-10.

32. Tashjian RZ, Levanthal E, Spenciner DB, Green A, Fleming BC. Initial fixation strength of massive rotator cuff tears: in vitro comparison of single-row suture anchor and transosseous tunnel constructs. Arthroscopy. 2007;23: 710-6.

33. Frank JB, ElAttrache NS, Dines JS, Blackburn A, Crues J, Tibone JE. Repair site integrity after arthroscopic transosseous-equivalent suture-bridge rotator cuff repair. Am J Sports Med. 2008:36:1496-503.

34. Behrens SB, Bruce B, Zonno AJ, Paller D, Green A. Initial fixation strength of transosseous-equivalent suture bridge rotator cuff repair is comparable with transosseous repair. Am J Sports Med. 2012;40:133-40.

35. Sano H, Yamashita T, Wakabayashi I, Itoi E. Stress distribution in the supraspinatus tendon after tendon repair: suture anchors versus transosseous suture fixation. Am J Sports Med. 2007;35:542-6.

36. Tuoheti Y, Itoi E, Yamamoto N, Seki N, Abe H, Minagawa H, et al. Contact area, contact pressure, and pressure patterns of the tendon-bone interface after rotator cuff repair. Am J Sports Med. 2005:33:1869-74.

37. Tingart MJ, Apreleva M, Lehtinen J, Zurakowski D, Warner JJP. Anchor design and bone mineral density affect the pull-out strength of suture anchors in rotator cuff repair: which anchors are best to use in patients with low bone quality? Am J Sports Med. 2004;32:1466-73.

38. Mahar A, Allred DW, Wedemeyer M, Abbi G, Pedowitz R. A biomechanical and radiographic analysis of standard and intracortical suture anchors for arthroscopic rotator cuff repair. Arthroscopy. 2006;22:130-5.

39. Apreleva M, Ozbaydar M, Fitzgibbons PG, Warner JJP, et al. Arthroscopy. 2002;18:519-26.

Ready to submit your research? Choose BMC and benefit from

- fast, convenient online submission

- thorough peer review by experienced researchers in your field

- rapid publication on acceptance

- support for research data, including large and complex data types

- gold Open Access which fosters wider collaboration and increased citations

- maximum visibility for your research: over $100 \mathrm{M}$ website views per year

At BMC, research is always in progress.

Learn more biomedcentral.com/submissions 